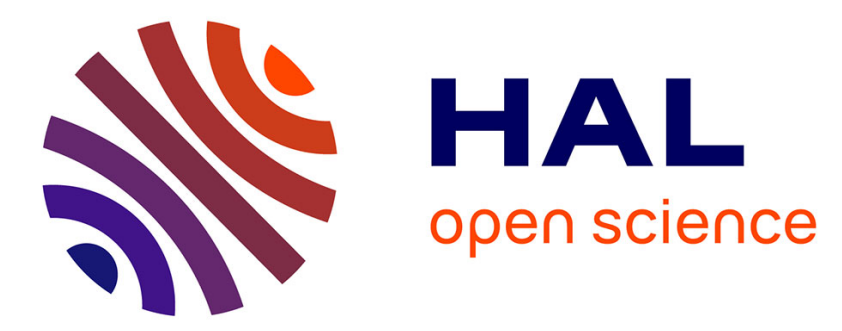

\title{
Computational passive imaging of thermal sources with a leaky chaotic cavity
}

Ariel Christopher Tondo Yoya, Benjamin Fuchs, Matthieu Davy

\section{To cite this version:}

Ariel Christopher Tondo Yoya, Benjamin Fuchs, Matthieu Davy. Computational passive imaging of thermal sources with a leaky chaotic cavity. Applied Physics Letters, 2017, 111 (19), pp.10.1063/1.4996964. 10.1063/1.4996964 . hal-01670962

HAL Id: hal-01670962

\section{https://hal-univ-rennes1.archives-ouvertes.fr/hal-01670962}

Submitted on 21 Jan 2021

HAL is a multi-disciplinary open access archive for the deposit and dissemination of scientific research documents, whether they are published or not. The documents may come from teaching and research institutions in France or abroad, or from public or private research centers.
L'archive ouverte pluridisciplinaire HAL, est destinée au dépôt et à la diffusion de documents scientifiques de niveau recherche, publiés ou non, émanant des établissements d'enseignement et de recherche français ou étrangers, des laboratoires publics ou privés. 


\title{
Computational passive imaging of thermal sources with a leaky chaotic cavity
}

\author{
Ariel Christopher Tondo Yoya, Benjamin Fuchs, Matthieu Davy* \\ Institute of Electronics and Telecommunications of Rennes, UMR CNRS 6164, University of Rennes \\ 1, 35042 Rennes, France
}

*Correspondence to: matthieu.davy@univ-rennes1.fr

\begin{abstract}
Computational imaging techniques are of great interest to simplify the architecture of imaging devices since random illuminations of a scene enable its reconstruction from few measurements by solving an inverse problem. Here we present a passive system for imaging of thermal sources in the microwave range from the cross-correlation of noise signals recorded by only two channels. The channels are attached to a high Q-factor chaotic cavity with a leaky aperture on its front side. The spatial distribution of noise sources is encoded onto the broadband spectrum of the cross-correlation and can be reconstructed from the sensing matrix mapping the uncorrelated far-field speckle patterns of the cavity onto a set of frequencies. We demonstrate imaging of localized and extended thermal sources and show that the polarization of those radiations can be discriminated. Moreover, we exhibit the effectiveness of the proposed system as a compressive imaging device which exploits the natural randomness of the speckle patterns. We believe that these results are a promising step for the design of real time and low cost microwave radiometers.
\end{abstract}

Computational imaging (CI) has received considerable attention in recent years. Instead of measuring the field on an array of detectors, the spatial information of an object or a scene is encoded onto independent illuminations collected by a single detector. Using the knowledge of the sensing matrix between the detector and the discretized volume of the scene for each illumination, the object can be reconstructed by solving an inverse problem. This makes it possible to strongly reduce the number of detectors and hence to design cheaper and faster imaging devices. In optics, the single pixel camera exploits the reflection of the incoming field on a spatial light modulator (SLM) for which independent coded projections are generated at a high frequency rate. ${ }^{1}$ Such a CI scheme has later been extended to the $\mathrm{THz}$ regime. ${ }^{2,3}$ A multiple scattering medium can also act as a compressive medium to limit the number of sensors. ${ }^{4}$

Similarly, the ghost imaging (GI) technique exploits spatially uncorrelated illuminations of an object to reconstruct its spatial distribution from measurements with a single detector. GI has originally been introduced with entangled photons ${ }^{5}$ and then generalized to classical light. ${ }^{6,7}$ An object illuminated with a spatially incoherent source is reconstructed from the aggregate correlation between the intensity collected with a bucket detector bearing no spatial resolution and a reference giving the intensity in the focal plane of the object. In classical GI, this reference is obtained by splitting the beam from a pseudothermal source and measuring the field that has not passed through the object with a multi-pixel camera. In computational GI, the independent illuminations are obtained by shaping the incoming field with pseudorandom patterns generated from a controlled SLM so that only a single detector is required..$^{8-}$ 
${ }^{10} \mathrm{GI}$ has demonstrated its ability to image objects in turbid media ${ }^{11,12}$ and has even been extended to $3 \mathrm{D}$ imaging. ${ }^{13-15}$ GI can also be combined with compressive sensing approaches to solve the inverse imaging problem from a reduced number of measurements by leveraging the sparsity of the scene. ${ }^{10,15-18}$

In acoustics ${ }^{19,20}$ and microwaves ${ }^{21-26}$, the spatial information can alternatively be encoded onto the spectrum of the broadband impulse response between an antenna and the scene. A highly dispersive medium such as a leaky chaotic cavity ${ }^{21-23}$, a metamaterial ${ }^{24,27}$ or a frequency diverse metasurface ${ }^{25,26,28}$ can be thought of as hardware sensing mechanisms for imaging. More specifically, the natural reverberation of the wave propagation inside the medium emulates random illuminations of a scene at different frequencies. These systems exploit the conversion between spatial and spectral degrees of freedom which are also at the core of the time reversal technique in complex media for spatio-temporal focusing with a single broadband antenna. ${ }^{29,30}$ The number of uncorrelated speckles over the bandwidth $B$ is $N_{\omega}=B \tau$, where $\tau$ is the typical decay time of the intensity within the cavity. Albeit random, the speckle patterns are deterministic and the far-field sensing operator $H(\omega, r)$ is obtained by scanning the near-field of the aperture of the medium and propagating it to the scene. An object can then be reconstructed from the measurement of a single spectrum without the need of an active control over an external device to modulate the incoming field.

Reducing the number of radiofrequency channels in radar imaging is of great interest to achieve realtime images. ${ }^{21}$ This is even more crucial for passive systems for which the distribution of thermal sources is reconstructed.$^{31}$ In the Rayleigh-Jeans limit ${ }^{32}$, the power spectral density of thermal radiations is given by $P=\epsilon k_{B} T_{a b s}$, where $\varepsilon$ is the emissivity, $T_{a b s}$ is the temperature and $k_{B}$ is the Boltzmann's constant. A synthetic aperture interferometric radiometer exploits measurements of the cross-correlation between noise signals simultaneously performed over each pairs of an array of receiving antennas. ${ }^{33,34}$ This creates a large aperture to obtain high resolution images. However, for ambient noise sources in the microwave range, the cross-correlation function has to be integrated over a long time to obtain a sufficient signal-to-noise ratio.

In this article, we demonstrate passive imaging of thermal sources in the $\mathrm{X}$-band frequency regime using a leaky chaotic cavity attached to two ports. We reconstruct the distribution of localized and extended thermal sources from the single cross-correlation of two recorded signals. Moreover, we show that the polarization of the sources can be discriminated and that the use of compressive sensing techniques improves significantly the reconstruction of sparse objects on a scene.

Thermal radiations are modeled by noise sources $n(r, \omega)$ with random distribution in a volume $V$. For the sake of simplicity, we consider a single polarization of thermal sources, the following formalism being similar for the other polarization. The signals recorded on two ports of a computational imaging system during an integration time $T$ are cross-correlated, $c_{12}(\tau)=\frac{1}{T} \int_{0}^{T} s_{1}(t) s_{2}(t+\tau) d t$. This gives equivalently in the frequency domain $c_{12}(\omega)=s_{1}(\omega) s_{2}^{*}(\omega)=$ $\iint_{V} H_{1}(\omega, r) H_{2}^{*}\left(\omega, r^{\prime}\right) n(r, \omega) n^{*}\left(r^{\prime}, \omega\right) d r d r^{\prime}$, where $H_{1,2}(\omega, r)$ are the sensing functions associated to port 1 and port 2, respectively. The noise correlation function in the Rayleigh-Jeans limit ${ }^{32}$ satisfies $\left\langle n(r, \omega) n^{*}\left(r^{\prime}, \omega^{\prime}\right)\right\rangle=k_{B} T_{e q}(r) \delta\left(\omega-\omega^{\prime}\right) \delta\left(r-r^{\prime}\right)$. Because thermal sources are spatially uncorrelated, the average cross-correlation gives for $T \rightarrow \infty$,

$$
c_{12}(\omega)=\int_{V} H(\omega, r) k_{B} T_{e q}(r) d r
$$

where $H(\omega, r)=H_{1}(\omega, r) H_{2}^{*}(\omega, r)$. The scene is discretized over $N$ pixels and sensed by $M$ measurements so that the matrix $\boldsymbol{H}$ is of dimension $M \times N$. Eq. (1) hence yields $\boldsymbol{c}_{\mathbf{1 2}}=\boldsymbol{H} \boldsymbol{x}$, with $x(r)=$ 
$k_{B} T(r)$. The reconstruction of the noise sources requires solving an inverse problem that can be formulated in the following generic way:

$$
\min _{\boldsymbol{x} \in \mathbb{R}^{+}}\|\boldsymbol{x}\|_{p} \text { subject to }\left\|\boldsymbol{c}_{\mathbf{1 2}}-\boldsymbol{H} \boldsymbol{x}\right\|_{2} \leq \delta .
$$

The unknown $\boldsymbol{x}$ is a real positive quantity (see Eq. (1)) and the parameter $\delta$ is a positive scalar to take into account inevitable additive noise due to finite integration time $T$ and measurement uncertainties on $\boldsymbol{H}$. In practice, the number of measurements is much smaller than the size of the scene to be reconstructed $(M \ll N)$ and the underdetermined linear problem admits many solutions. The role of the regularizer $\|x\|_{p}$, that stands for the $\ell_{p}$-norm of the vector $\boldsymbol{x}$, is to foster a solution that best corresponds to the likely composition of the scene to reconstruct.

The solution $x(r)$ of Eq. (2) is unique only when the rank of $\boldsymbol{H}$ is equal or greater than $N$. The efficiency of the chaotic cavity imaging system as a compressive sensing setup is therefore linked to its ability to scramble the information received from its leaky aperture to the ports. We use an aluminum cavity with outer dimensions of $50 \times 50 \times 30 \mathrm{~cm}^{3}$ (see Fig. 1a). The ports are two coaxial to waveguide transitions 16094-SF40 attached to two sides of the cavity. The cavity is made chaotic by adding inside three metal hemispheres of radius $50 \mathrm{~mm}$ on the walls and a deformed corner. An array of $11 \mathrm{x} 11$ holes are perforated on the front face of the cavity covering an area of $34 \times 34 \mathrm{~cm}^{2}$. The diameter of the holes is chosen to be $\lambda_{\min } / 2=12.5 \mathrm{~mm}$, where $\lambda_{\min }$ is the average wavelength corresponding to the higher frequency. This ensures a good tradeoff between the overall Q-factor of the cavity and the radiated power. ${ }^{22}$ This is crucial to detect small amplitude noise sources in a passive scenario.

Using a waveguide transition translated over a plane at a distance of $5 \mathrm{~mm}$ from the aperture, we first scan the near-field of the cavity on a regular grid of $N^{\prime}=756$ positions with steps of $\lambda_{\min } / 3$. The nearfield transmitted fields $h_{1,2}\left(r_{n}, \omega\right)$ are measured on $M=4001$ frequencies in steps of $1 \mathrm{MHz}$ from 8 to $12 \mathrm{GHz}$ using a vectorial network analyzer. The scan is successively carried out for vertical and horizontal polarizations of the probe. Due to multiple reflections within the cavity, the two polarizations are uncorrelated and statistically equivalent. The amplitude and phase of a single spectrum are shown in Fig. 1b. The reconstruction of the matrices $\boldsymbol{H}_{1,2}$ on the plane of thermal sources is obtained by projecting the near field speckle patterns on the image pixels using the dyadic Green's functions for each polarization, $H_{1,2}(\omega, r)=\sum_{n=1}^{N \prime} h_{1,2}\left(r_{n}, \omega\right) G\left(r_{n}, r, \omega\right)$, where $h_{1,2}\left(r_{n}, \omega\right)$ are the near-field scans of the cavity on positions $r_{n}$ for an excitation from ports 1 and 2, respectively, and $G\left(r_{n}, r, \omega\right)$ is the free space Green's function between $r_{n}$ and $r$. Speckle patterns of $H(\omega, r)$ at three frequencies are presented in Fig. 1d.

The matrix of correlation coefficients between near-field patterns at different frequencies is seen in Fig. $1 \mathrm{c}$ to be nearly diagonal. By computing the spectral field-field correlation function, we find that the frequency spacing above which two speckle patterns transmitted through the aperture are statistically independent is $\delta f=3 \mathrm{MHz}$. We therefore estimate the spectral degrees of freedom to be $N_{\omega} \sim 1137$ over the bandwidth. The corresponding quality factor of the cavity is equal to $Q=2840$ at $10 \mathrm{GHz}$.

To demonstrate imaging of localized noise sources, we use horn antennas connected to distinct amplifiers with high noise temperature and terminated with $50 \Omega$ loads. The antennas therefore radiate independent noise signals generated by the amplifiers. The signals $s_{1}(t)$ and $s_{2}(t)$ at the ports are downconverted to the [0-4] GHz range with a mixer and a local oscillator at $8 \mathrm{GHz}$ and are recorded with a digital sampling oscilloscope with a bandwidth of $6 \mathrm{GHz}$ and a sampling rate of $10 \mathrm{GS} / \mathrm{s}$. 
Equation (2) is first solved using the popular Tikhonov regularization, case of the $\ell_{2}$-norm, which is known to improve the conditioning of the problem while providing a direct numerical solution. The results are shown for a single source and two sources in Fig. 2a,b respectively. The resolution $\delta_{a}$ is determined by the cavity aperture $D$. As in synthetic aperture radar imaging, it can be estimated by $\delta_{a}=$ $\lambda_{\min } F / D$, where $F$ is the distance to the target. This is in agreement with the resolution found experimentally as shown in Supplementary Material.

We then explore the signal to noise ratio (SNR) as a function of the number of frequencies used to reconstruct the image. Similarly to the GI technique, ${ }^{9,35}$ the SNR is seen in Fig. 2c to increase as the square root of the number of random illuminations, $\sqrt{M}$, for $M<N_{\omega}$. The SNR saturates from $M>N_{\omega}$ since the speckle patterns become slightly correlated. The quality of the reconstruction also increases as $\sqrt{T}$, where $T$ is the integration time of the cross-correlation, and reaches a plateau for $T \sim 100 \mu$ s (see Fig. 2d) as a consequence of the maximum number $N_{\omega}$.

To further enhance the SNR, another strategy consists in leveraging the inherent sparsity of most natural imaged scenes. The sources are indeed typically located at some particular range in specific directions. A convenient mean to foster sparse solutions is to use a $\ell_{1}$-norm regularizer, $\|x\|_{1}$, as now widely used since the seminal works of Donoho, Candès, Tao and Romberg. ${ }^{36-38}$ They demonstrated that a perfect reconstruction can be achieved with high probability from a small number of measurements when the sensing matrix $\boldsymbol{H}$ is a random matrix. In the frame of compressive sensing with a chaotic cavity, this means that a sparse scene can be properly imaged from the measurement of $c_{12}(\omega)$ at only a small number of frequencies using $\ell_{1}$ minimization. In Fig. 3a, we present the reconstruction of the two sources using this sparse promoting regularizer and $M=N_{\omega}$. The sources appear at two pixels in perfect agreement with the result of Tikhonov regularization.

In Fig. 3b, we show the probability of successful reconstruction over 20 trials as a function of the number of sources $K$ and the number of frequencies $M$ randomly chosen from the set of the $N_{\omega}$ independent frequencies. To this end, we first record individually the cross-correlation for 16 locations of the horn antenna. The cross-correlation $c_{12}(\omega)$ is then computed synthetically for a given $K$ by summing $K$ signals. The solution of Eq. (2) with the $\ell_{1}$-norm regularizer is compared to the image of reference found for $M=N_{\omega}$. The reconstruction is assumed to be successful when the correlation coefficient between the reconstructed and reference images of $N=676$ pixels is higher than 0.8 .

A sharp transition is observed between unsuccessful and successful recovery. This transition is reminiscent of the phase transition introduced by Donoho and Tanner in compressive sensing. ${ }^{39}$ When the entries of the sensing matrix $\boldsymbol{H}$ are statistically independent variables with identical Gaussian distribution, a perfect reconstruction from undersampled measurements can indeed be achieved with only $O(K \log (N / K))$ measurements. For $K=12$, the transition is found experimentally at $M \sim 400$, which is higher than the theoretical level. We explain this increase by the errors inherent to the construction of the sensing matrix $\boldsymbol{H}$ from the near-field scan of the aperture. Indeed, simulations with an exact knowledge of $\boldsymbol{H}$ yields a transition at $M \sim 100$ as expected in theory.

Finally, to further prove the capabilities of our system for extended thermal sources, we use two commercial straight fluorescent lamps (FL) of length $60 \mathrm{~cm}$ as thermal sources located at a distance $F=$ $0.4 \mathrm{~m}$ from the aperture of the cavity. FL behave as broadband microwave noise sources ${ }^{40}$ with a temperature which is approximately the electron temperature of the gas discharge. ${ }^{41,42}$ Those thermal sources are mainly polarized perpendicularly to their axis. FL that are turned on indeed behave as metallic reflectors for an incident wave polarized along their axis. ${ }^{43}$ This is confirmed by measuring the intensity transmitted and reflected by four vertical FL turned off and on for the two polarizations. 
Kirchhoff's law of thermal radiation states that the emissivity $\varepsilon$ of an object is equal to its absorptivity $\alpha$. We estimate $\varepsilon \sim 0.2$ and $\varepsilon \sim 0.75$ for vertical and horizontal polarizations, respectively.

We first aim to image two FL that are vertically positioned along y-axis with a spacing of $11 \mathrm{~cm}$. The recorded signals are amplified with two low noise amplifiers (LNA) with a gain of $51 \mathrm{~dB}$ within the [812] GHz frequency range and an equivalent temperature of $67 \mathrm{~K}$. Because the noise emitted by the LNAs are also radiated by the ports inside the cavity, this yields a coherent contribution to the cross-correlation function. We mitigate this unwanted contribution by subtracting the noise cross-correlation in absence of the FL. We solve Eq. (2) with a sensing matrix corresponding successively to the horizontal and vertical polarizations. The reconstruction of two vertical FL from the horizontally polarized sensing matrix is shown in Fig. 4a for an integration time of $T=1 \mathrm{~ms}$. The two FL clearly appear at horizontal positions $0.07 \mathrm{~m}$ and $0.18 \mathrm{~m}$. In contrast the use of the vertically polarized sensing matrix yields a noisy image (see Fig. 4b). The tubes are then rotated and a clear reconstruction of the FL is obtained only using the sensing matrix computed from the vertically polarized scan (see Fig. 4c,d). This result demonstrates the capacity of our system to image extended thermal sources and discriminate their polarization with only two detectors.

In conclusion, we have achieved experimentally the reconstruction of localized and extended thermal sources in the microwave range with only two ports of a leaky chaotic cavity using computational imaging and compressive sensing techniques. We also have demonstrated that our system makes it possible to separate the polarization of thermal sources. This work opens the door to the design of radiometers with real-time imaging capabilities of great interest for medical imaging, remote sensing or security screening applications. Further studies will be dedicated to demonstrate that it is even possible to achieve passive imaging from the auto-correlation of the noise signal at a single port yielding an intensity-only imaging system. ${ }^{44}$

This publication is supported by the European Union through the European Regional Development Fund (ERDF) and by the french region of Brittany and Rennes Métropole through the CPER Project SOPHIE / STIC \& Ondes. The authors would also like to acknowledge Cécile Leconte for her help in automating the scan. 


\section{FIGURES}

(a)

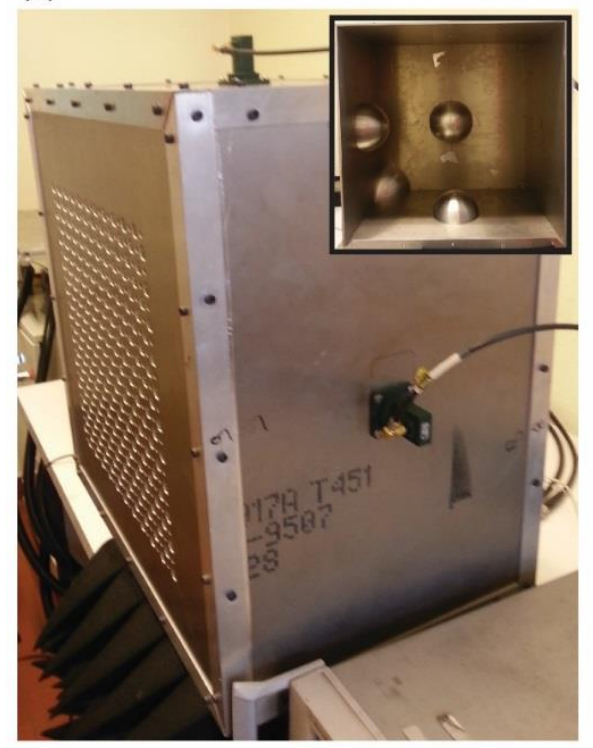

(b)

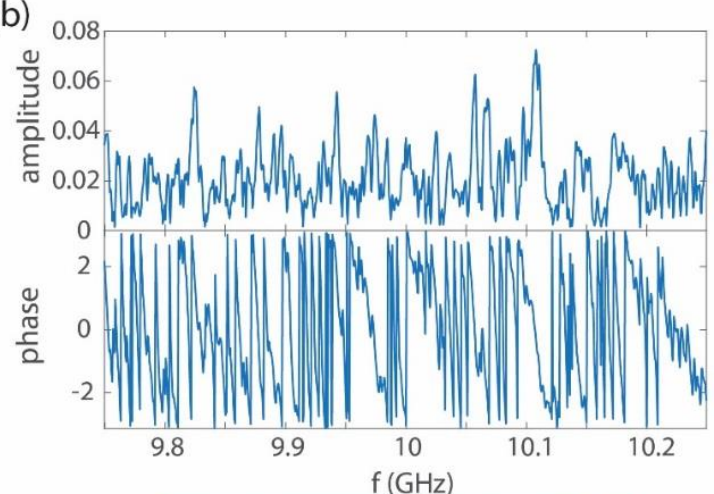

(c)

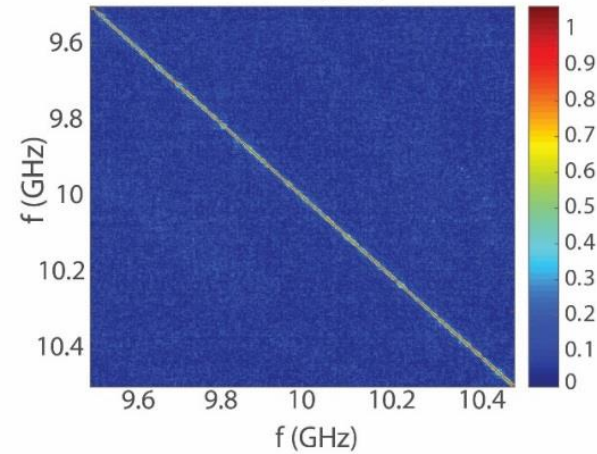

(d)

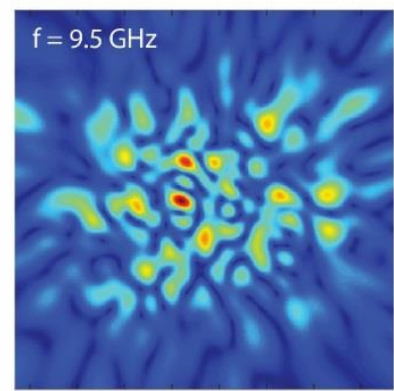

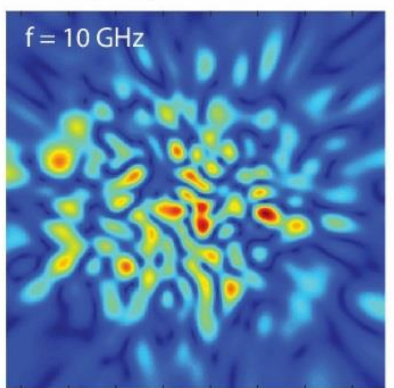

Fig 1. (a) Photography of the leaky cavity with an aperture made of drilled holes on its front side. Inset: Interior of the chaotic cavity. (b) Amplitude and phase of a single transmitted field from port 1 through the aperture. (c) Spectral field correlation matrix between 9.5 and $10.5 \mathrm{GHz}$. (d) Three speckle patterns at $\mathrm{f}=9.5 \mathrm{GHz}, \mathrm{f}=9.75 \mathrm{GHz}$ and $\mathrm{f}=10 \mathrm{GHz}$ at a distance $F=0.5 \mathrm{~m}$ from the aperture computed from the propagation of the near-field scan of the cavity. 

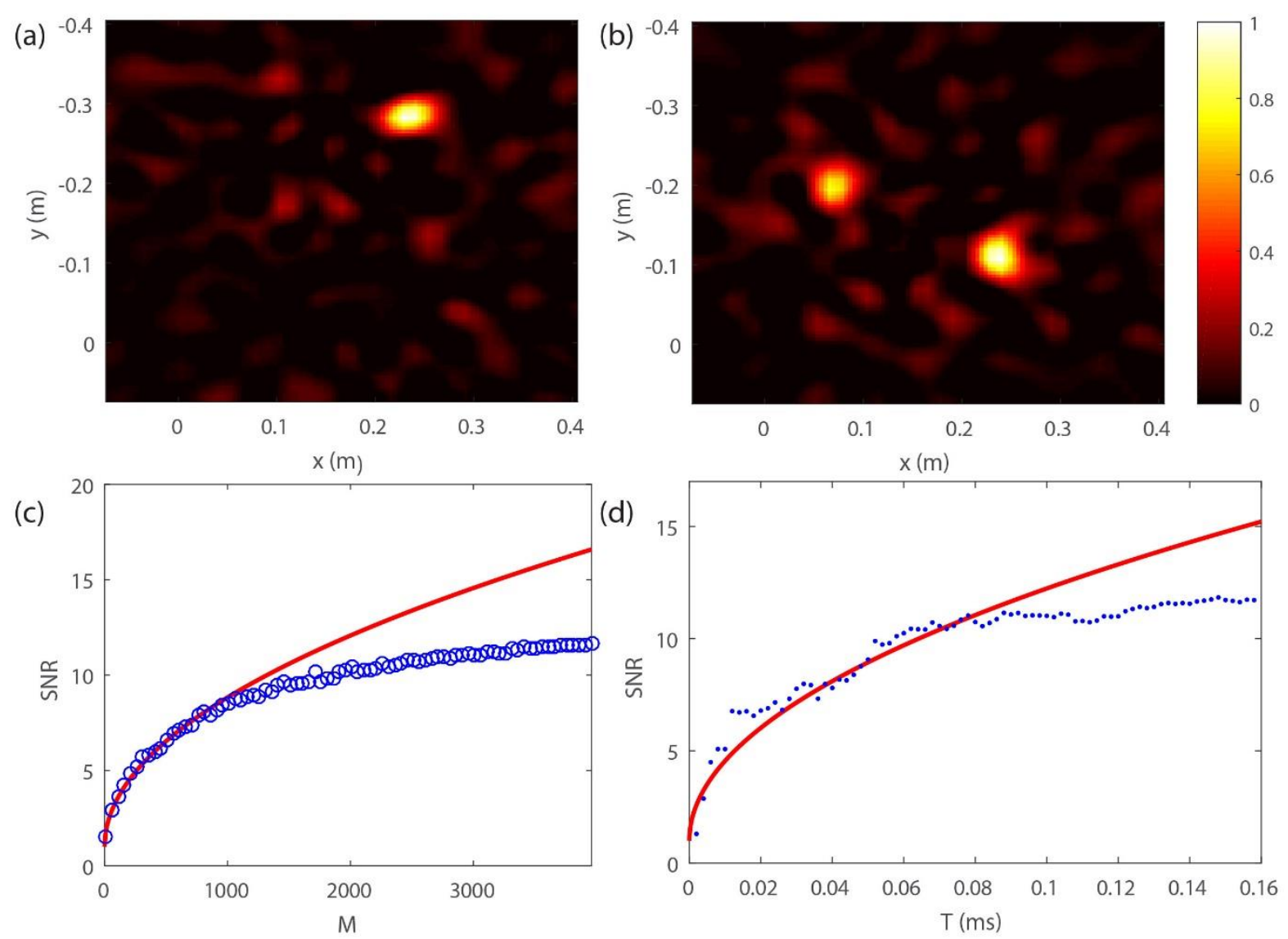

Fig. 2: (a,b) Reconstruction of one (a) and two (b) noise sources at a distance $F=0.5 \mathrm{~m}$ from the aperture of the cavity. (c) SNR as a function of the number of frequencies $M$ used to reconstruct the image. The circles are measurements and the red line shows $\sqrt{M}$ dependence (d) SNR as a function of the integration time of the cross-correlation $T$. Experimental results (blue dots) are compared to an increase as $\sqrt{T}$.
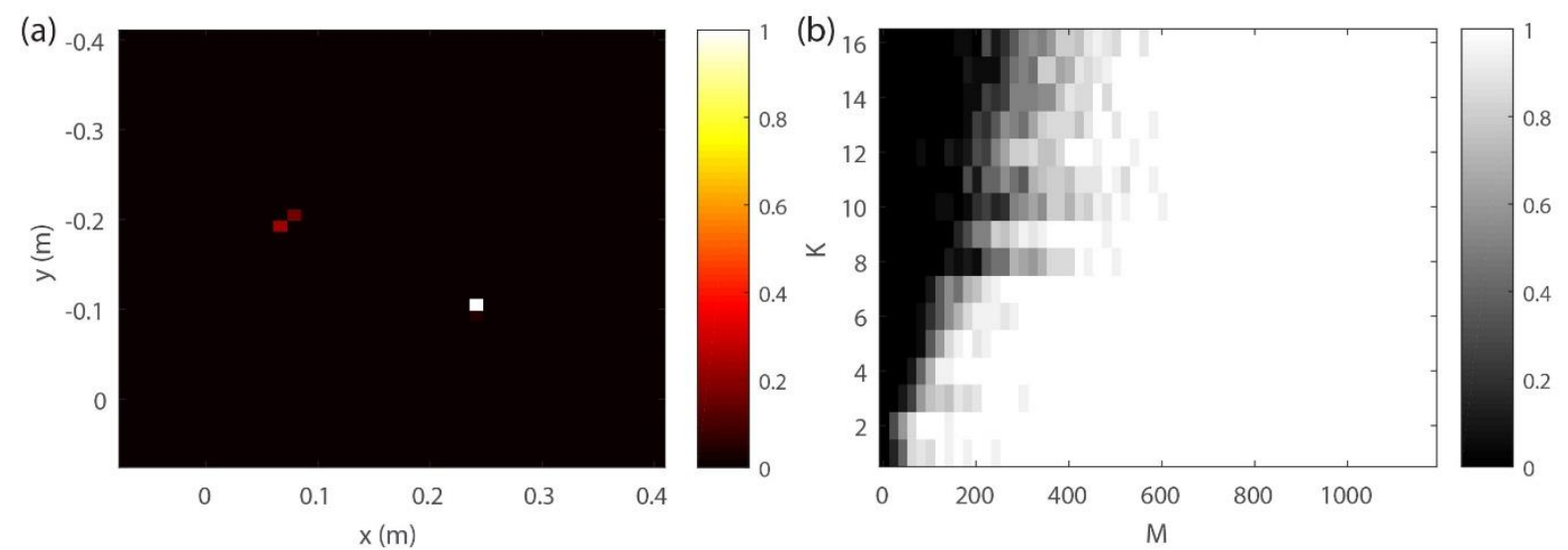

Fig. 3: (a) Reconstruction of two noise sources by solving Eq. (2) using a sparse recovery approach $\left(\ell_{1}\right.$ regularization). (b) Probability of success recovery between 0 and 1 for $K$ noise sources ( $K$ between 1 and 10) for $N=676$ pixels. The scale indicates the percentage of successful recovery from $M$ measurements of the sparse scene. A perfect recovery is achieved in the white region. 

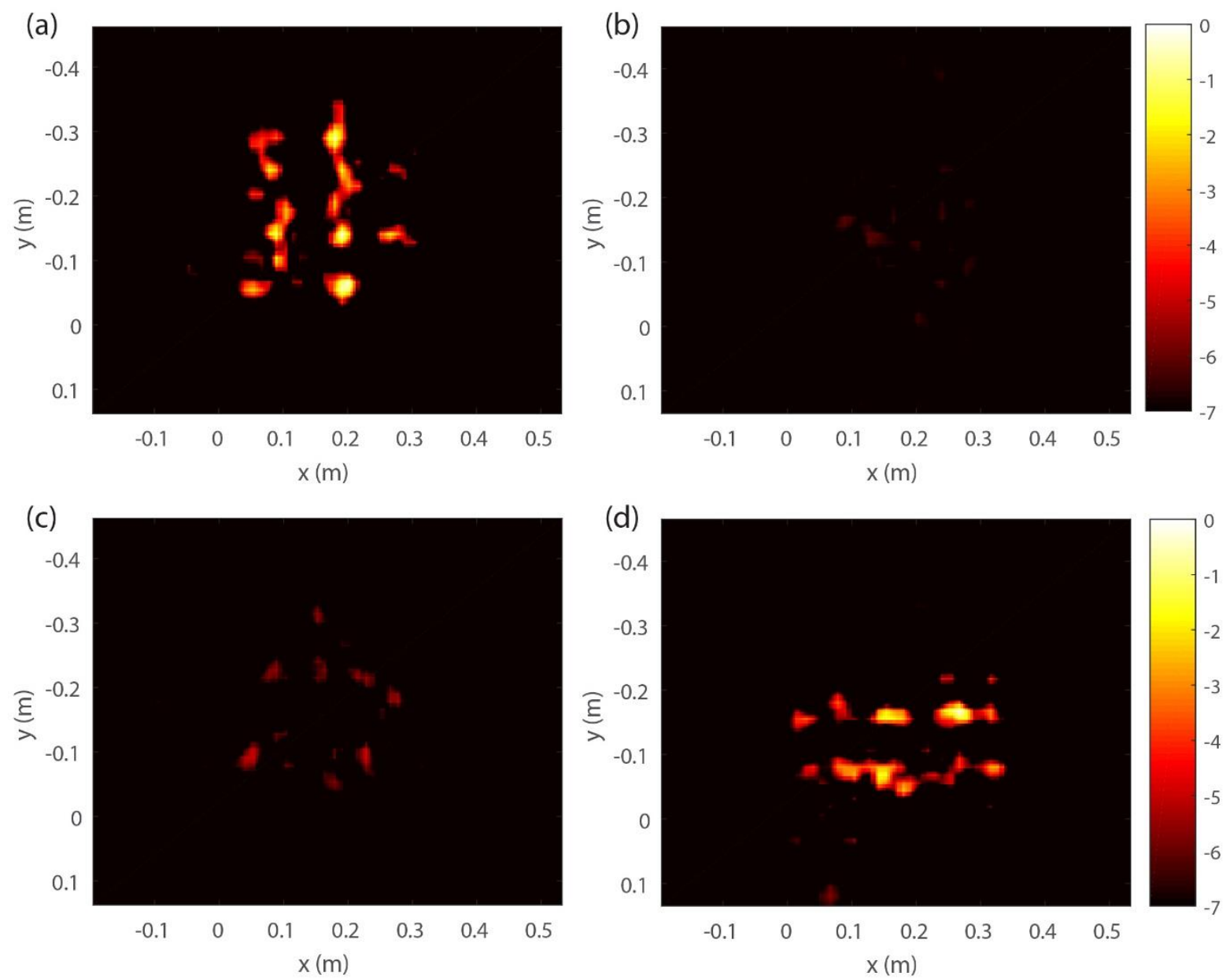

Fig. 4: Reconstruction of broadband extended thermal sources which are two straight fluorescent lamps spaced by $0.11 \mathrm{~m}$ and located at a distance $F=0.4 \mathrm{~m}$ from the aperture of the cavity. (a,b) The tubes are positioned along the y-axis and the image is computed from the horizontal (a) and vertical polarizations (b). (c,d) the tubes are positioned along the $\mathrm{x}$-axis and the image is computed from the horizontal (c) and vertical (d) polarizations.

\section{REFERENCES}

M. F. Duarte, M. A. Davenport, D. Takhar, J. N. Laska, T. Sun, K. E. Kelly, and R. G. Baraniuk, IEEE Signal Process. Lett. 25 (2), 83 (2008).

2 W. L. Chan, K. Charan, D. Takhar, K. F. Kelly, R. G. Baraniuk, and D. M. Mittleman, Appl. Phys. Lett. 93 (12), 121105 (2008).

3 C. M. Watts, D. Shrekenhamer, J. Montoya, G. Lipworth, J. Hunt, T. Sleasman, S. Krishna, D. R. Smith, and W. J. Padilla, Nat. Photon. 8 (8), 605 (2014).

4 A. Liutkus, D. Martina, S. Popoff, G. Chardon, O. Katz, G. Lerosey, S. Gigan, L. Daudet, and I. Carron, Sci. Rep. 4 (2014). 
T. B. Pittman, Y. H. Shih, D. V. Strekalov, and A. V. Sergienko, Phys. Rev. A 52 (5), R3429 (1995).

A. Valencia, G. Scarcelli, M. D’Angelo, and Y. Shih, Phys. Rev. Lett. 94 (6), 063601 (2005).

F. Ferri, D. Magatti, A. Gatti, M. Bache, E. Brambilla, and L. A. Lugiato, Phys. Rev. Lett. 94 (18), 183602 (2005).

J. H. Shapiro, Phys. Rev. A 78 (6), 061802 (2008).

Y. Bromberg, O. Katz, and Y. Silberberg, Phys. Rev. A 79 (5), 053840 (2009).

M. Aßmann and M. Bayer, Sci. Rep. 3, 1545 (2013).

M. Bina, D. Magatti, M. Molteni, A. Gatti, L. A. Lugiato, and F. Ferri, Phys. Rev. Lett. 110 (8), 083901 (2013).

R. E. Meyers, K. S. Deacon, and Y. Shih, Appl. Phys. Lett. 98 (11), 111115 (2011).

N. D. Hardy and J. H. Shapiro, Phys. Rev. A 87 (2), 023820 (2013).

B. Sun, M. P. Edgar, R. Bowman, L. E. Vittert, S. Welsh, A. Bowman, and M. Padgett, Science 340 (6134), 844 (2013).

W. Gong, C. Zhao, H. Yu, M. Chen, W. Xu, and S. Han, Sci. Rep. 6, 26133 (2016).

O. Katz, Y. Bromberg, and Y. Silberberg, Appl. Phys. Lett. 95 (13), 131110 (2009).

P. Zerom, K. W. C. Chan, J. C. Howell, and R. W. Boyd, Phys. Rev. A 84 (6), 061804 (2011).

P. A. Morris, R. S. Aspden, J. E. C. Bell, R. W. Boyd, and M. J. Padgett, Nat. Comm. 6, 5913 (2015).

G. Montaldo, D. Palacio, M. Tanter, and M. Fink, IEEE Trans. Ultrason., Ferroelect., Freq. Control 52 (9), 1489 (2005).

Y. Xie, T.-H. Tsai, A. Konneker, B.-I. Popa, D. J. Brady, and S. A. Cummer, Proc. Natl. Acad. Sci. 112 (34), 10595 (2015).

T. Fromenteze, C. Decroze, and D. Carsenat, IEEE Trans. Antennas Propag. 63 (2), 593 (2015). T. Fromenteze, O. Yurduseven, M. F. Imani, J. Gollub, C. Decroze, D. Carsenat, and D. R. Smith, Appl. Phys. Lett. 106 (19), 194104 (2015).

O. Yurduseven, V. R. Gowda, J. N. Gollub, and D. R. Smith, IEEE Microw. Wirel. Compon. Lett. 26 (5), 367 (2016).

J. Hunt, T. Driscoll, A. Mrozack, G. Lipworth, M. Reynolds, D. Brady, and D. R. Smith, Science 339 (6117), 310 (2013).

O. Yurduseven, J. N. Gollub, A. Rose, D. L. Marks, and D. R. Smith, IEEE Access 4, 5436 (2016).

J. N. Gollub, O. Yurduseven, K. P. Trofatter, D. Arnitz, M. F. Imani, T. Sleasman, M. Boyarsky, A. Rose, A. Pedross-Engel, H. Odabasi, T. Zvolensky, G. Lipworth, D. Brady, D. L. Marks, M. S. Reynolds, and D. R. Smith, Sci. Rep. 7, 42650 (2017).

G. Lipworth, A. Mrozack, J. Hunt, D. L. Marks, T. Driscoll, D. Brady, and D. R. Smith, J. Opt. Soc. Am. A 30 (8), 1603 (2013).

T. Zvolensky, J. N. Gollub, D. L. Marks, and D. R. Smith, IEEE Access PP (99), 1 (2017).

M. Fink, Phys. Today 50 (3), 34 (1997).

F. Lemoult, G. Lerosey, J. de Rosny, and M. Fink, Phys. Rev. Lett. 103 (17), 173902 (2009).

E. L. Kpré and C. Decroze, IEEE Geosci. Remote Sens. Lett. PP (99), 1 (2017).

R. H. Dicke, Rev. Sci. Instrum. 17 (7), 268 (1946).

C. S. Ruf, C. T. Swift, A. B. Tanner, and D. M. L. Vine, IEEE Trans. Geosci. Remote Sens. 26 (5), 597 (1988).

D. M. L. Vine, A. J. Griffis, C. T. Swift, and T. J. Jackson, Proc. IEEE 82 (12), 1787 (1994).

B. I. Erkmen and J. H. Shapiro, Phys. Rev. A 79 (2), 023833 (2009).

D. L. Donoho and M. Elad, Proc. Natl. Acad. Sci. 100 (5), 2197 (2003).

E. J. Candès, J. K. Romberg, and T. Tao, Comm. Pure Appl. Math. 59 (8), 1207 (2006).

D. L. Donoho, IEEE Trans. Inf. Theory 52 (4), 1289 (2006).

D. L. Donoho and J. Tanner, Proc. IEEE 98 (6), 913 (2010).

W. W. Mumford, Bell Syst. Tech. J. 28 (4), 608 (1949).

M. A. Easley and W. W. Mumford, J. Appl. Phys. 22 (6), 846 (1951).

E. Maxwell and B. J. Leon, IRE Trans. Microw. Theory Techn. 4 (2), 81 (1956).

M. Laroussi and J. R. Roth, IEEE Transactions on Plasma Science 21 (4), 366 (1993). 

(2016). 